\title{
(3) Fluorescent Silica Nanoparticles with Multivalent Inhibitory Effects towards Carbonic Anhydrases
}

\author{
Nadia Touisni, ${ }^{[a]}$ Nasreddine Kanfar, ${ }^{[a]}$ Sébastien Ulrich, ${ }^{[a]}$ Pascal Dumy, ${ }^{[a]}$ Claudiu T. Supuran, ${ }^{[b]}$ \\ Ahmad Mehdi, ${ }^{[c]}$ and Jean-Yves Winum*[a]
}

\begin{abstract}
Multifunctional silica nanoparticles decorated with fluorescent and sulfonamide carbonic anhydrase (CA) inhibitors were prepared and investigated as multivalent enzyme inhibitors against the cytosolic isoforms hCA I and II and the transmembrane tumor-associated ones hCA IX and XII. Excellent inhibitory effects were observed with these nanoparticles, with $K_{\mathrm{I}}$ values in the low nanomolar range $(6.2-0.67 \mathrm{~nm})$ against all tested isozymes. A significant multivalency effect was seen for the inhibition of the monomeric enzymes hCA I and II compared to the dimeric hCA IX and hCA XII isoforms, where no multivalent effect was observed, suggesting that the multivalent binding is occurring through enzyme clustering.
\end{abstract}

Carbonic anhydrases (CAs, EC 4.2.1.1) are well known zinc metalloproteins which catalyze a simple but essential physiological reaction: carbon dioxide hydration to bicarbonate and proton. ${ }^{[1]}$ These enzymes are of clinical relevance in cancer therapy as, among the 15 isoforms known in humans, some catalytically active isozymes are critical in various fundamental physiological processes and have been clinically exploited for the treatment or prevention of various pathologies such as glaucoma and neurological disorders. The lack of selectivity of conventional inhibitors against the different CA isozymes have led researchers to investigate new approaches for achieving such a goal. ${ }^{[1]}$ For example, different strategies were reported in recent years for obtaining compounds that specifically target the tumor-associated CA IX ${ }^{[2]}$ especially the sugar approaches developed by our group. ${ }^{[3,4]}$ Beside the rational drug

[a] Dr. N. Touisni, N. Kanfar, Dr. S. Ulrich, Prof. Dr. P. Dumy, Dr. J.-Y. Winum Institut des Biomolécules Max Mousseron (IBMM)

UMR 5247 CNRS-ENSCM-Université de Montpellier, ENSCM

8 rue de l'Ecole Normale, 34296 Montpellier Cedex (France) E-mail:jean-yves.winum@univ-montp2.fr

[b] Prof. Dr. C. T. Supuran

Neurofarba Department

Section of Pharmaceutical and Nutriceutical Sciences

Università degli Studi di Firenze

Via Ugo Schiff 6, 50019 Sesto Fiorentino, Florence (Italy)

[c] Prof. Dr. A. Mehdi

Institut Charles Gerhardt

UMR 5253, Equipe Chimie Moléculaire et Organisation du Solide

Université de Montpellier

Place Eugène Bataillon, 34095 Montpellier Cedex 05 (France)

Supporting information for this article is available on the WWW under http://dx.doi.org/10.1002/chem.201501037. design of more selective enzyme inhibitors, the nanoparticles approach was applied by our group to carbonic anhydrases and we demonstrated the possibility of activity modulation of carbonic anhydrases using nano-objects. ${ }^{[5,6]}$ Due to their size, ease of synthesis, and their potential applications, silica-based nanoparticles received great attention for their biomedical applications both for the site-specific delivery of drugs or for imaging purposes. ${ }^{[7-9]}$ Moreover it constitutes a good platform for developing multivalent interactions, which is a promising option for the specific treatment of diseases.

Multivalent enzyme inhibitors that combine multiple copies of an inhibitor conjugated to a single scaffold may lead to an overall increase of the binding affinity, avidity, and/or specificity. ${ }^{[10]}$ These changes that are associated with the multivalent presentation of inhibitors depend on the nature of the biological target and may arise from different mechanisms: 1) a statistical rebinding effect, 2) a chelate effect, 3) a subsite binding effect, or 4) a clustering effect. ${ }^{[11]}$ Though the multivalency approach has been extensively studied in the context of lectins that contain multiple binding sites, ${ }^{[12]}$ its application in the field of enzyme inhibition has received less attention until recently. For instance, the multivalency strategy has been successfully applied in the case of glycosidase inhibitors. ${ }^{[13-14]}$ In the case of carbonic anhydrase, only studies on bivalent inhibitors were recently reported. Indeed, Whiteside's research group described in 2012 the binding of monovalent and bivalent benzene sulfonamide ligands to a synthetic dimer of carbonic anhydrase. ${ }^{[15]}$ The group of Neri reported the use of bivalent small molecule ligand-drug conjugates against carbonic anhydrase IX. ${ }^{[16]}$ Besides the classical small-molecule approach, which has been applied with some success on the in vitro and in vivo inhibition of human CA (hCA), the use of multivalent nanoplatforms could therefore be of great interest and a promising strategy in this field.

In this paper, we describe the synthesis and multivalent inhibition of sulfonamide carbonic anhydrase inhibitor-coated silica nanoparticles against cytosolic/transmembrane CA isoforms.

CA inhibitor-coated silica nanoparticles were prepared following the synthetic strategy depicted in Scheme 1. First, two organosilylated ligands $\mathbf{3}$ and $\mathbf{6}$ were synthesized, the first one by coupling 3-aminopropyltriisopropoxylsilane 1 with fluorescein isothiocyanate $\mathbf{2}$, the latter by coupling of 3-aminopropyltriisopropylsilane 1 with 4-isothiocyanato(ethyl) benzene sulfonamide 5. Previously, 6 has been prepared by reacting 4aminoethylbenzene sulfonamide $\mathbf{4}$ with dicyclohexylcarbodi- 


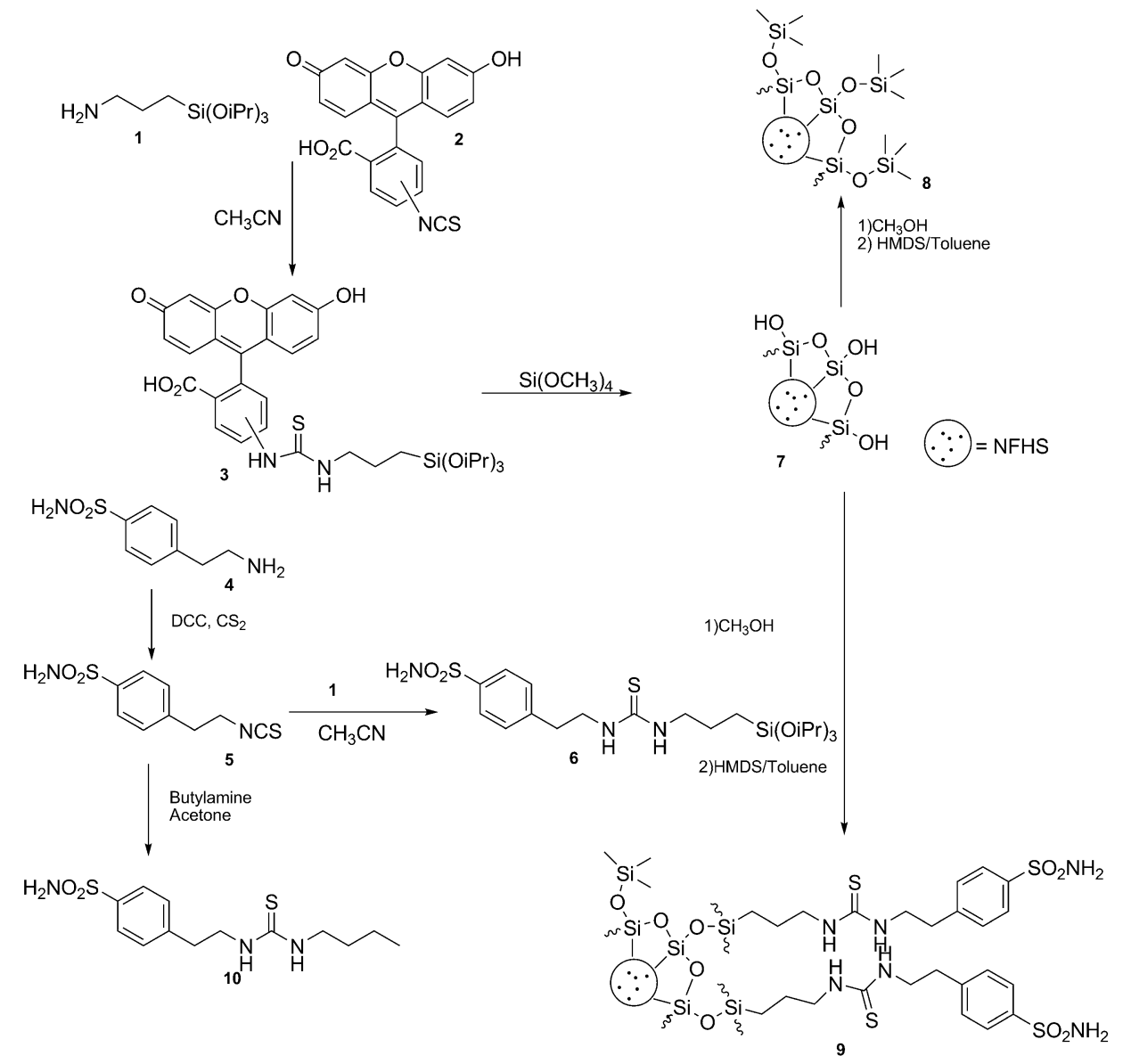

Scheme 1. Schematic representation of NFHS particle synthesis with carbonic anhydrase inhibitors immobilization. particles. The results showed that the particles 9 were monodisperse with a diameter of about ( $3.5 \pm 0.8) \mathrm{nm}$ (Figure 1).

The nitrogen sorption analyses showed that the NFHS particles are not porous, and the Brunauer-Emmett-Teller (BET) specific surface areas are respectively $330 \mathrm{~m}^{2} \mathrm{~g}^{-1}$ for NFHS particle 7 and $260 \mathrm{~m}^{2} \mathrm{~g}^{-1}$ for NFHS particle 9. This diminution shows clearly the surface modification by the ligand 6 . With the help of elemental analyses, a silica/ligand molar ratio of around 250 was obtained for nanoparticle 9. Based on NFHS particle size and this ratio, the number of sulfonamide ligands per nanoparticle was estimated to be between 12 and 14 (see the Supporting Information).

The NFHS particles 8 and $\mathbf{9}$ have been assayed for their inhibitory potency against the physiologically relevant cytosolic hCA I and II, as well as against the two tumour-associated isoforms hCA IX and XII. It can be observed from the data in Table 1 that no inhibition or a weak inhibition was observed

imide (DCC) and carbon disulfide. ${ }^{[17,18]}$ Isothiocyanate $\mathbf{5}$ was reacted with $n$-butylamine in acetone to afford the thiourea 10 , which was used as a monovalent reference in the enzymatic inhibition assays. The nanometer-sized fluorescent hybrid silica (NFHS) particles 7 were then prepared by a hydrolysis and polycondensation approach, using the water-in-oil microemulsion method. ${ }^{[19-21]}$ Co-hydrolysis and polycondensation of a mixture of the fluorescent silylated precursor 3 and tetramethoxysilane in the presence of a base as catalyst in an ultrasound bath $^{[22]}$ led to fluorescein-labelled nanoparticle 7, which was isolated after centrifugation and several treatments with ethanol and water. The silica surface was then modified, on the one hand, by treatment with methanol overnight under reflux. The capping of $\mathrm{Si}-\mathrm{OH}$ functions was performed by treatment with hexamethyldisilazane (HMDS) for four hours to yield the nanoparticle $\mathbf{8}^{\left[{ }^{[23]}\right.}$ On the other hand, the bifunctional (fluorescent + inhibitor) system was prepared by reacting nanoparticle 7 with silylated inhibitor ligand 6 in methanol under reflux overnight. The same treatment with HMDS was subsequently performed to afford nanoparticle 9 (Scheme 1).

The size of the composite NFHS particles was investigated by transmission electron microscopy (TEM) and the mean diameter and standard derivation of the particles were determined from TEM pictures by averaging the size of 150

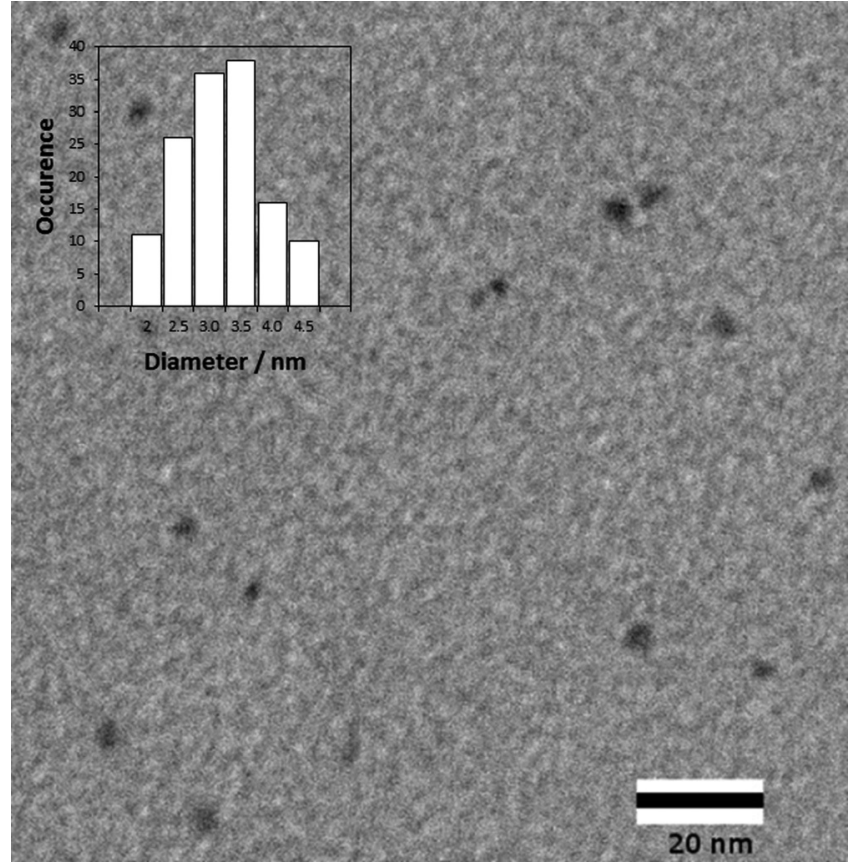

Figure 1. TEM picture of NFHS particle 9. 


\begin{tabular}{|c|c|c|c|c|}
\hline & \multicolumn{4}{|c|}{$K_{1}[\mathrm{~nm}]^{[a]}$} \\
\hline & hCA I & hCA II & hCA IX & hCA XII \\
\hline 10 & 142 & 48.1 & 38.3 & 29.8 \\
\hline 8 & 38800 & 268 & 560 & 92650 \\
\hline 9 & 4.4 & 0.67 & 2.7 & 6.2 \\
\hline $\mathrm{rp}$ & 32.3 & 71.8 & 14.2 & 4.8 \\
\hline $\mathrm{rp} / \mathrm{n}$ & 2.7 & 6 & 1 & 0.4 \\
\hline
\end{tabular}

[a] Mean from three different assays, errors in the range of $\pm 10 \%$ of the reported values (data not shown). rp: relative potency $=K_{1}(\mathbf{1 0}) / K_{1}(\mathbf{9})$. $\mathrm{rp} / \mathrm{n}$ : relative potency/number of sulfonamide units.

for all isoforms with the inhibitor-free nanoparticle 8. However, nanoparticle 9 exhibited excellent inhibitory effects, in low nanomolar range, with $K_{\mathrm{l}}$ values in the range $6.2-0.67 \mathrm{~nm}$ against all tested isozymes.

Multivalent effects were clearly seen for nanoparticle $\mathbf{9}$ when compared with monovalent inhibitor 10 . With hCA I and II, the inhibition constants $K_{1}$ decrease from 142 to $4.4 \mathrm{nM}$ for hCA I and from $48.1 \mathrm{nM}$ to $670 \mathrm{pM}$ for hCA II, which corresponds to an inhibitory potency improvement of 32 and 72 times, respectively (Table 1). Interestingly, no multivalent effect was characterized in the case of hCA IX, where a linear inhibitory effect was observed in reference to the ligand 10 , the relative potency normalized to the sulfonamide units $(\mathrm{rp} / n)$ being equal to 1 . For the isoform XII, an increase of the overall inhibitory potency from 29.8 to $6.2 \mathrm{~nm}$ was observed between the monovalent compound 10 and the multivalent system 9 , but amounted to a negative multivalent effect; the relative potency of a sulfonamide inhibitor being weaker in the nanoplatform compared to the single compound reference. These results indicate that the multivalency approach can be a promising strategy for enhancing not only the binding affinity of CA inhibitors but also the selectivity between different isoforms. For instance, the selectivity was modified when shifting from a monovalent $\left(K_{\mathrm{I}}(\mathrm{hCA} I \mathrm{I}) / K_{\mathrm{I}}(\mathrm{hCA} \mathrm{IX})=1.25\right)$ to a multivalent system $\left(K_{\mathrm{I}}(\mathrm{hCA} I \mathrm{I}) /\right.$ $\left.K_{l}(\mathrm{hCA} I X)=0.24\right)$, the monovalent inhibitor becoming a hCA IIselective multivalent inhibitor compared to hCA IX and hCA XII.

Note also the inhibition data observed with inhibitor-free nanoparticles 8. Even if inhibitory activity is relatively weak, a significant selectivity was noticed with hCA II and hCA IX compared to other isoforms tested. The inhibitory mechanism could be explained by adsorption phenomena of silica nanoparticles on the border of the active site cavity. ${ }^{[25]}$

In conclusion, NFHS nanoparticles incorporating fluorescein and benzenesulfonamide moieties were prepared by an original procedure in order to investigate the effect of multivalency on the inhibition of CA isoforms with important physiologic and pathologic functions such as the widely distributed cytosolic CA I and $I I^{[26]}$ as well as the transmembrane, tumorassociated CA IX and XII. ${ }^{[27]}$ In fact, the only other contribution dealing with this was our earlier work in which we reported gold nanoparticles derivatized with a thiol-containing benzenesulfonamide, which showed excellent inhibitory and multiva- lency effects for inhibiting the tumor-associated CA IX (but were much less effective as CA I and II inhibitors). ${ }^{[5]}$ For the nanoparticles reported here, based on silica nanoparticles, the opposite effect was observed: for the cytosolic isoforms hCA I and II, the inhibition constants decreased from 142 to $4.4 \mathrm{nM}$ for hCA I, and from 48 to $0.67 \mathrm{~nm}$ for hCA II, corresponding to an inhibitory potency improvement of 32 and 72 times, respectively, when compared to the monovalent system. However, multivalency effects were not observed for the transmembrane isoforms hCA IX and XII. The main difference between the cytosolic isoforms hCA I and II on the one hand, and hCA IX and $\mathrm{XII}$ on the other hand (apart from the different cellular localization) is that the two transmembrane isoforms are dimers, whereas the cytosolic CA I and II are monomers. ${ }^{[28,29]}$ Although the active sites of CA I, II, IX and XII are rather similar, the fact that the two transmembrane isoforms have two different active sites in their molecule may have consequences for the access of a multivalent inhibitor in both of them concomitantly. In fact, the sulfonamide $\mathbf{1 0}$ is a better inhibitor of hCA IX and XII compared to hCA I and II. However, this effect is not preserved when 10 was attached to the NFHS nanoparticles. However, a significant multivalency effect was seen for the inhibition of hCA I and II (monomeric enzymes) probably because there are no steric hindrance effects related to the access of the derivatized nanoparticle to the enzyme active site. This may suggest that multivalent binding occurs in this case through enzyme clustering. ${ }^{[1]}$ As hCA I and II are important drug targets for obtaining diuretics, ${ }^{[30]}$ antiglaucoma, ${ }^{[31]}$ and anticonvulsant agents, ${ }^{[32]}$ we estimate that this type of NFHS nanoparticle may have interesting biomedical theragnostic applications, combining therapy through carbonic anhydrase inhibition and fluorescence imaging.

\section{Acknowledgements}

This work was supported by the French Ministry of Research (MESR) with a Ph.D fellowship for N.K. We thank the CNRS and LabEx CheMISyst (ANR-10-LABX-05-01) and La Ligue contre le Cancer (Comité des Pyrénées-Orientales) for funding. Authors thank Simon Cassegrain for technical assistance designing the cover page.

Keywords: carbonic anhydrase • inhibitors • multivalency nanoparticles $\cdot$ sulfonamide

[1] C. T. Supuran, Nat. Rev. Drug Discovery 2008, 7, 168-181.

[2] J.-Y. Winum, M. Rami, A. Scozzafava, J. L. Montero, C. T. Supuran, Med. Res. Rev. 2008, 28, 445-463.

[3] J.-Y. Winum, S. A. Poulsen, C. T. Supuran, Med. Res. Rev. 2009, 29, 419435.

[4] J.-Y. Winum, P. A. Colinas, C. T. Supuran, Bioorg. Med. Chem. 2013, 21, $1419-1426$.

[5] M. Stiti, A. Cecchi, M. Rami, M. Abdaoui, A. Scozzafava, Y. Guari, J.-Y. Winum, C. T. Supuran, J. Am. Chem. Soc. 2008, 130, 16130-16131.

[6] M. C. Saada, J. L. Montero, D. Vullo, A. Scozzafava, J.-Y. Winum, C. T. Supuran, J. Med. Chem. 2011, 54, 1170-1177.

[7] K. E. Sapsford, W. R. Algar, L. Berti, K. B. Gemmill, B. J. Casey, E. Oh, M. H. Stewart, I. L. Medintz, Chem. Rev. 2013, 113, 1904-2074. 
[8] S. Iltis, J. Choi, M. Vollmers, M. Shenoi, J. Bischof, G. J. Metzger, NMR Biomed. 2014, 27, 1063-1069.

[9] M. Helle, E. Rampazzo, M. Monchanin, F. Marchal, F. Guillemin, S. Bonacchi, F. Salis, L. Prodi, L. Bezdetnaya, ACS Nano 2013, 7, 8645-8657.

[10] C. Fasting, C. A. Schalley, M. Weber, O. Seitz, S. Hecht, B. Koksch, J. Dernedde, C. Graf, E.-W. Knapp, R. Haag, Angew. Chem. Int. Ed. 2012, 51 10472-10498; Angew. Chem. 2012, 124, 10622-10650.

[11] L. L. Kiessling, J. E. Gestwicki, L. E. Strong, Angew. Chem. Int. Ed. 2006 45, 2348-2368; Angew. Chem. 2006, 118, 2408-2429.

[12] O. Renaudet, R. Roy, Chem. Soc. Rev. 2013, 42, 4515-4517.

[13] P. Compain, A. Bodlenner, ChemBioChem 2014, 15, 1239-1251.

[14] S. G. Gouin, Chem. Eur. J. 2014, 20, 11616-11628.

[15] E. T. Mack, P. W. Snyder, R. Perez-Castillejos, B. Bilgiçer, D. T. Moustakas, M. J. Butte, G. M. Whitesides, J. Am. Chem. Soc. 2012, 134, 333- 345.

[16] N. Krall, F. Pretto, W. Decurtins, G. J. L. Bernardes, C. T. Supuran, D. Neri, Angew. Chem. Int. Ed. 2014, 53, 4231-4235; Angew. Chem. 2014, 126, 4315-4320.

[17] S. Eiden-Assmann, B. Lindlar, G. Maret, J. Colloid Interface Sci. 2004, 271, $120-123$.

[18] K. S. Rao, K. El-Hami, T. Kodaki, K. Matsushige, K. Makino, J. Colloid Interface Sci. 2005, 289, 125-131.

[19] W. Yang, C. G. Zhang, H. Y. Qu, H. H. Yang, J. G. Xu, Anal. Chim. Acto 2004, 503, $163-169$.

[20] W. Tan, K. Wang, X. He, X. J. Zhao, T. Drake, L. Wang, R. P. Bagwe, Med. Res. Rev. 2004, 24, 621-638.
[21] R. P. Bagwe, C. Yang, L. R. Hilliard, W. Tan, Langmuir 2004, 20, 8336 8342

[22] H. H. Yang, H. Y. Ou, P. Lin, S. H. Li, M. T. Ding, J. G. Xu, Analyst 2003 $128,462-466$.

[23] The capping of the $\mathrm{Si}-\mathrm{OH}$ group was realized in order to reduce drastically the interaction of silanol functions with the surface of the protein, and reduce the potential inhibitory activity of non-capped nanoparticles.

[24] R. G. Khalifah, J. Biol. Chem. 1971, 246, 2561-2573.

[25] M. Karlsson, L.-G. Martensson, B.-H. Jonsson, U. Carlsson, Langmuir 2000, 16, 8470-8479.

[26] C. T. Supuran, J. Enzyme Inhib. Med. Chem. 2012, 27, 759-772.

[27] F. Allouche, F. Chabchoub, F. Carta, C. T. Supuran, J. Enzyme Inhib. Med. Chem. 2013, 28, 343-349.

[28] C. T. Supuran, J. Enzyme Inhib. Med. Chem. 2013, 28, 229-230.

[29] D. Neri, C. T. Supuran, Nat. Rev. Drug Discovery 2011, 10, 767-777.

[30] F. Carta, C. T. Supuran, Expert Opin. Ther. Pat. 2013, 23, 681-691.

[31] F. Fabrizi, F. Mincione, T. Somma, G. Scozzafava, F. Galassi, E. Masini, F. Impagnatiello, C. T. Supuran, J. Enzyme Inhib. Med. Chem. 2012, 27, $138-147$.

[32] C. T. Supuran, Expert Opin. Ther. Pat. 2013, 23, 677-679.

Received: March 17, 2015

Published online on May 12, 2015 